\title{
BMJ Open Effect of Tai Chi for the prevention or treatment of osteoporosis in elderly adults: protocol for a systematic review and meta-analysis
}

Wei-qiang Mu, Xia-yu Huang, Jiang Zhang, Xiao-cong Liu, Mao-mao Huang

To cite: Mu W, Huang $X$, Zhang J, et al. Effect of Tai Chi for the prevention or treatment of osteoporosis in elderly adults: protocol for a systematic review and meta-analysis. BMJ Open 2018:8:e020123. doi:10.1136/ bmjopen-2017-020123

- Prepublication history and additional material for this paper are available online. To view these files, please visit the journal online (http://dx.doi. org/10.1136/bmjopen-2017020123).

W-M and X-H contributed equally.

Received 24 October 2017 Revised 6 March 2018 Accepted 12 March 2018
Check for updates

Jingdezhen City Hospital of Traditional Chinese Medicine, Jingdezhen, China

Correspondence to

Mao-mao Huang;

huangmaoma088@sina.com

\section{ABSTRACT}

Introduction 0steoporosis (OP) has been defined as a degenerative bone disease characterised by low bone mass and microstructural deterioration of bone tissue, leading to fragility and an increased risk of fractures, especially of the hip, spine and wrist. Exercise has been shown to benefit the maintenance of bone health and improvement of muscle strength, balance and coordination, thereby reducing the risk of falls and fractures. However, prior findings regarding the optimal types and regimens of exercise for treating low bone mineral density (BMD) in elderly people are not consistent. As an important component of traditional Chinese Qigong exercises, Tai Chi (TC) is an ancient art and science of healthcare derived from the martial arts. The objective of this study is to attempt to conduct a systematic review and meta-analysis of the existing studies on TC exercise as an intervention for the prevention or treatment of $\mathrm{OP}$ in elderly adults and to draw more useful conclusions regarding the safety and the effectiveness of TC in preventing or treating OP.

Methods and analysis Eight electronic databases (Science Citation Index, PubMed Database, Embase (Ovid) Database, the Cochrane Central Register of Controlled Trials, and Chinese databases, including Chinese BioMedical Database, China National Knowledge Infrastructure, Wanfang database and the Chongqing VIP Chinese Science and Technology Periodical Database) will be searched from the beginning of each database to 1 April 2018. Potential outcomes of interest will include rates of fractures or falls, BMD at the total hip and the total spine, bone formation biomarkers, bone resorption biomarkers, bone biomarkers, health-related quality of life and adverse events. Only randomised controlled trials comparing TC exercise against each other or nonintervention will be included. The Cochrane risk of bias assessment tool will be used for quality assessment. Ethics and dissemination Ethical approval is not required as the study will be a review of existing studies. This review may help to elucidate whether TC exercise is effective for the prevention or treatment of OP in elderly adults. The findings of the study will be published in a peer-reviewed publication and will be disseminated electronically or in print. We will share the findings in the fourth quarter of 2018.

Trial registration number CRD42018084950.
Strengths and limitations of this study

- A comprehensive search of existing Chinese databases will be conducted from their respective inceptions to date.

- The quality of the included studies, all randomised controlled trials, is strongly warranted.

- The study screening, data extraction and risk of bias assessment will be completed independently by two reviewers and any discrepancy will be resolved by discussion or will be decided by a third reviewer.

- The current study is a protocol for a systematic review and meta-analysis without data analysis results, which will be conducted after the protocol.

- Our results may be limited by heterogeneity due to differences in age, gender, geographic region, provoking agent and the heterogeneity of study designs.

\section{INTRODUCTION}

Osteoporosis (OP) has been defined as a degenerative bone disease characterised by low bone mass and microstructural deterioration of bone tissue, leading to fragility and an increased risk of fractures, especially of the hip, spine and wrist. ${ }^{1}$ According to the United States National Osteoporosis Foundation, $\mathrm{OP}$ is a quiet epidemic and a major threat to public health. In the USA, Europe and Japan, approximately 75 million people suffer from OP. ${ }^{2}$ Although OP affects both sexes, $80 \%$ of those who have OP are women. ${ }^{3}$ OP affects quality of life, especially an individual's physical state of health, a loss of free movement and possible fractures ${ }^{4}$ and causes significant morbidity and mortality in the elderly. Furthermore, the prevalence of OP increases with age and portends an increase in caregiver burden. OP is costly and requires complicated medical care and nursing home placement and is an economic burden both for the individual and for society. These impacts will be even more pronounced in the future because of the demographic shift, 
resulting in an increased number of elderly citizens. ${ }^{5}$ As a result, it is of substantial importance to detect optimised prevention or treatment for OP. Since drug therapy is an expensive option with uncertain consequences and may be associated with adverse effects including arthralgia myalgia instance or fever, ${ }^{6}$ non-pharmacological therapy offers an attractive alternative. ${ }^{7}$ One of the most effective prevention strategies for the prevention or treatment of OP is exercise. ${ }^{8}$ Exercise has been shown to benefit in the maintenance of bone health and improvement of muscle strength, balance and coordination, ${ }^{9}$ thereby reducing the risk of falls and fractures. A number of randomised controlled trials (RCTs) have shown that exercise can decrease age-related losses in bone mineral density (BMD) at the hip and spine in elderly adults. ${ }^{10-12}$ However, documented evidence of the optimal types and regimens of exercise for treating low BMD in elderly people is not consistent.

As an important component of traditional Chinese Qigong exercises, Tai Chi (TC) is an ancient art and science of healthcare derived from the martial arts. To date, TC has developed into several styles, including 'Wu', 'Yang', 'Chen' or 'Sun' styles, which features gentle, smooth, coordinated and flowing movements of different body parts accompanied by deep breathing and mental concentration. ${ }^{13}$ As an exercise for helping the healthy, TC has attracted considerable attention in the rehabilitation and geriatric community both in China and in Western countries. ${ }^{14}$ A substantial number of studies have reported that TC has been considered safe and effective for treating low BMD, promoting bone health and reducing the risk of fracture. ${ }^{15-18} \mathrm{At}$ present, systematic reviews on TC for the prevention or treatment of OP still depend on literature before 2008. Furthermore, the results from the only two systematic reviews examining TC for OP are in English and have inconsistent results. The 2007 systematic review by Wayne et al including six controlled studies, suggested that TC-naive women who participated in TC training exhibited reduced rates of postmenopausal declines in $\mathrm{BMD},{ }^{19}$ but the 2008 review by Lee et al including five RCTs, and two controlled clinical trials indicated that no significant effect of TC on BMD changed at the spine compared with no treatment in postmenopausal women. ${ }^{20} \mathrm{~A}$ recent systematic review by Chang et al in 2014 suggested that TC had a significant and positive effect on balance, ${ }^{21}$ but the evidence for the direct effects of TC on BMD was not reviewed. In addition, the evidence for TC on the prevention or treatment of OP is not convincing because of limited rigorous research. The objective of the current study is to attempt to conduct a systematic review and meta-analysis of the existing studies on TC exercise as an intervention for the prevention or treatment of OP in elderly adults to draw more useful conclusions about the safety and the effectiveness of TC in preventing or treating OP.

\section{OBJECTIVES}

This study is designed to conduct a systematic assessment of the effectiveness and safety of TC for the prevention and treatment of OP in elderly adults.

\section{METHODS AND ANALYSIS}

This systematic review protocol is, where appropriate, in line with the Preferred Reporting Items for Systematic Review and Meta-Analysis (PRISMA) Protocols checklist. ${ }^{22}$

\section{Eligibility criteria \\ Participants}

The target population was elderly adults aged 60 years or older with no distinction of sex or ethnicity, diagnosed with or without OP (as diagnosed using any recognised diagnostic criteria).

\section{Patient and public involvement}

This is the protocol for a systematic review and there is no patient and public involvement.

\section{Interventions}

Only RCTs comparing TC exercise against others, such as sedentary lifestyle, exercise, pharmacological treatment or calcium supplements, will be included. Any type of TC exercise will be included, regardless of the exercise frequency and duration.

\section{Outcomes}

Potential outcomes of interest are at least among the following:

\section{Primary outcomes}

1. Rates of OP-related fractures or falls;

2. BMD at the total hip and the total spine;

3. Bone formation biomarkers, such as serum bone-specific alkaline phosphatase and osteocalcin;

4. Bone resorption biomarkers, such as serum and urinary pyridinoline, type I collagen cross-linked N-telopeptides and C-telopeptides;

5. Bone biomarkers, such as parathyroid hormone and 1.25- $(\mathrm{OH}) 2$ vitamin $\mathrm{D}_{3}$;

6. Calcium concentrations in the serum and urine of study subjects.

\section{Secondary outcomes}

1. Health-related quality of life measurements using validated tools, such as Euroqol-5D $\mathrm{D}^{23}$ or the Medical Outcomes Study 36-item Short Form Health Survey ${ }^{24}$;

2. Adverse effects: any adverse events associated with the use of TC for treatment or prevention.

\section{Information sources and literature search}

Electronic database searches will be conducted using the following databases: Science Citation Index, PubMed Database, Embase (Ovid) Database, the Cochrane Central Register of Controlled Trials (Cochrane Library, current issue) and four Chinese databases will be searched, 


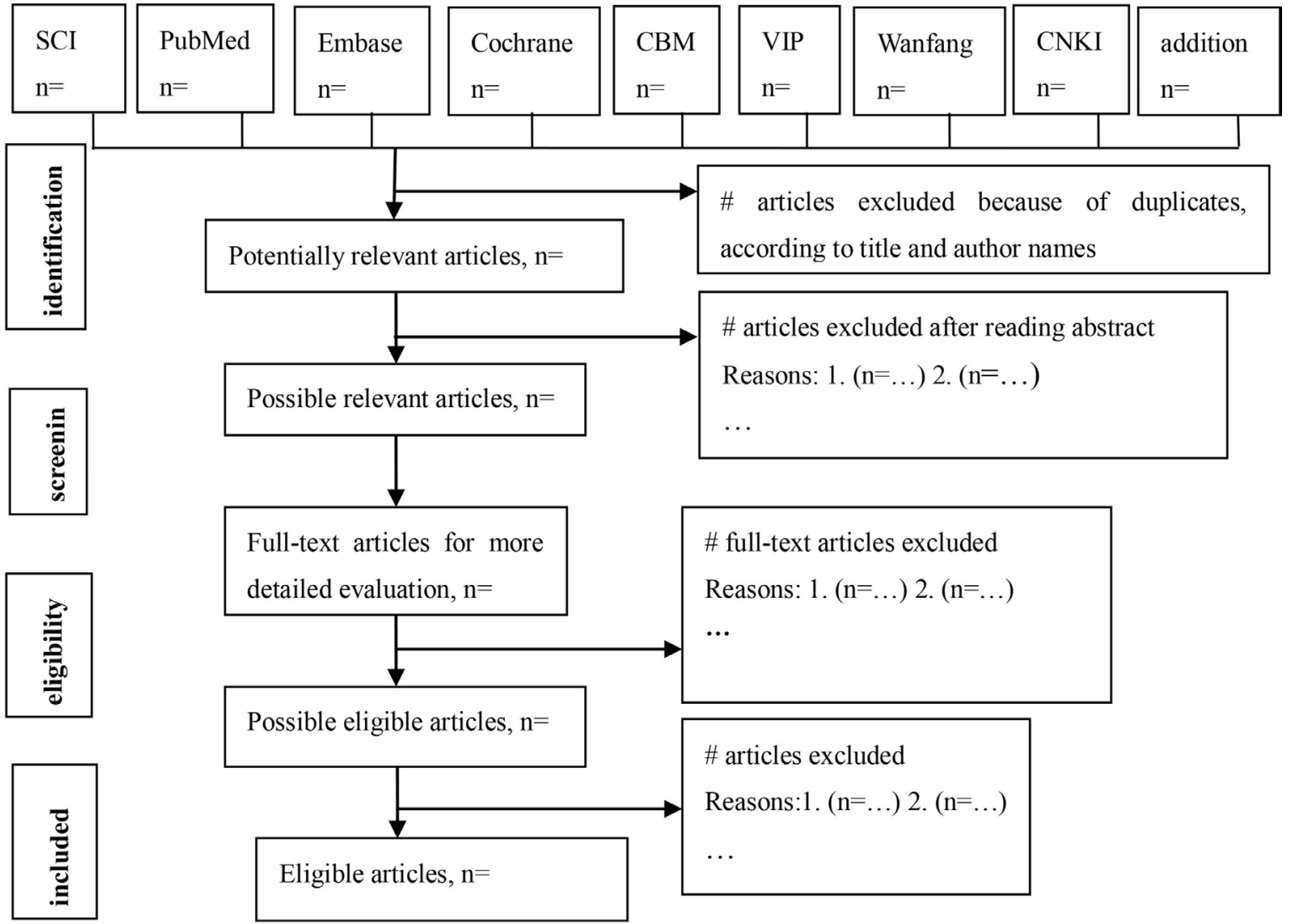

Figure 1 Flow chart of study selection. CBM, Chinese BioMedical Database; CNKI, China National Knowledge Infrastructure; SCI, Science Citation Index; VIP, Chongqing VIP Chinese Science and Technology Periodical Database.

namely, the Chinese BioMedical Database, the China National Knowledge Infrastructure, the Chongqing VIP Chinese Science and Technology Periodical Database and Wanfang Database. Each database will be searched from the beginning to 1 April 2018. In addition, the references of all relevant reviews will be hand-searched for further relevant studies. The search strategies will be developed using medical subject headings and text words related to the prevention or treatment of OP in older adults. The search terms will include "Tai Ji," "bone mineral density" and "osteoporosis." The detailed search strategy in each database can be found in the online supplementary file, and search results will be presented in a meta-analyses (PRISMA) flow diagram. ${ }^{25}$ Due to time and resource limitations, only English and Chinese studies will be included and Chinese studies will be translated into English.

\section{Study selection process}

Studies will be selected when (1) the design was an RCT; (2) the study population was elderly adults aged 60 years or older; (3) one of the interventions was a form of TC; (4) relevant outcomes such as rates of OP-related fractures or falls, BMD or bone biomarkers were assessed. Two authors (WQM and MMH) will review all of the titles and abstracts yielded in the search against the inclusion criteria independently and in duplicate to assess the eligibility of the searched studies. The full text of the articles and the relevant references that pass the first eligibility screening will be obtained to make a final selection of the studies included for the review. Any conflicts of study identification will be resolved by discussion, for example, through plenary meetings or discussions by email or will be decided by a third reviewer $(\mathrm{XYH})$. If necessary, we will contact the study author for additional information regarding the study. In addition, the reasons for study exclusion will be documented. A flow chart of study selection is shown in figure 1 .

\section{Data items and data collection process}

For studies fulfilling the inclusion criteria, data extraction will be completed independently by two reviewers (MMH and XCL) using a simple, standardised form that will describe the study characteristics. The primary headings will include basic information, population characteristics, methodological description, intervention characteristics and both baseline, postintervention and follow-up outcomes. The detailed data extraction form will include the following:

- Basic information (study design, title, name of authors, publication status, publication date, country, sample size, source of funding);

- Population characteristics (number of participant, mean age, proportion of each gender, with or without $\mathrm{OP}$, diagnostic criteria of $\mathrm{OP}$ if reported); 
- Methodological description (method of randomisation, method of concealment of allocation, blinding, losses to follow-up);

- Intervention characteristics (type and period of intervention, routes of administration, profession delivering the intervention);

- Outcome data (rates of OP-related fractures or falls, BMD, bone biomarkers, quality of life).

Extracted data will be checked by WQM and any discrepancy will be resolved by discussion or will be decided by a third reviewer $(\mathrm{XYH})$.

\section{Methodological quality/risk of bias appraisal}

All risk of bias of the included studies assessments will be performed independently by two reviewers (MMH, $\mathrm{XCL}$ ), with disagreements being resolved by discussion or a third reviewer (JZ) if necessary. Reviewers will not assess risk of bias of studies on which they were coauthors. We will apply the Cochrane Risk of Bias Assessment Tool which assesses against six potential sources of bias (adequate sequence generation, concealment of allocation, blinding of outcomes, adequately addressed incomplete outcome data, free from selective reporting and other sources of bias) ${ }^{26}$ and rate each criterion for each study as a low risk of bias, a high risk of bias or an unclear risk of bias. Overall, the quality assessment will consider the following aspects:

- Adequate sequence generation: whether the allocation sequence was generated appropriately;

- Concealment of allocation: whether study participants and research staff were unaware of the intervention given at the enrolment stage;

- Blinding of outcomes: whether the personnel assessing outcomes and analysing the data were blinded to the intervention allocated;

- Adequately addressed incomplete outcome data: were incomplete outcome data adequately reported in the published study?

- Free from selective reporting: whether the outcomes were reported selectively;

- Other sources of bias: whether the study was apparently free of any other high risk of bias (such as funding and potential for conflict of interest).

\section{Data synthesis and meta-analysis}

Information summarising the characteristics and results of the included studies will be summarised in a table named 'Characteristics of included studies' supplemented by a narrative summary that will evaluate the methods used and results among the studies. ${ }^{25}$ The summary table will include study identification (author and year), the country of publication, methods, the number of participants ( $\mathrm{n}$ and \%), mean age, type of intervention, frequency and duration of intervention and outcomes. In addition, the studies will be grouped depending on whether they are diagnosed with OP, and the information will be exported to Review Manager Software V.5.2 (2011, Cochrane Collaboration and Updated Software).
Meta-analysis will be performed only using studies regarded as suitably homogeneous by Review Manager Software V.5.2. If a meta-analysis is not possible, the results from clinically comparable trials will be performed descriptively. ${ }^{26}$ Effect sizes expressed as relative risks with 95\% CIs for categorical variables, and mean differences (if possible) or standardised mean differences (if necessary due to different measurement scales) for continuous data with $95 \%$ CIs will be reported. Heterogeneity of each outcome will be assessed statistically using the $\chi^{2}$ test $^{27}$ and Higgins $\mathrm{I}^{2}$ statistic. ${ }^{28}$ If there is no heterogeneity $(>0.1)$, a fixed effects model will be presented in meta-analysis, otherwise a random effects model will be applied. If significant statistical heterogeneity (ie, $<0.1$ or I $^{2}$ statistic $\left.>60 \%\right)^{29}$ are detected, meta-regressions and subgroup analyses will be further explored after first considering the following factors: study population and methodology, intervention and statistical heterogeneity).

\section{Reporting of the review}

The results of the systematic review will be reported in accordance with the PRISMA guidelines. ${ }^{30}$

\section{Confidence in cumulative evidence}

The quantitative outcomes will be summarised in a 'Summary of findings' table. In this case, each undesirable impact on our outcomes will be assessed using an approach based on the Grading of Recommendations Assessment, Development and Evaluation. ${ }^{31}$ The assessments will be categorised as high, moderate, low and very low quality.

Acknowledgements The authors would especially like to thank Zheng Guo-hua, $\mathrm{PhD}$ for editing this manuscript.

Contributors $\mathrm{XYH}$ designed the study protocol and drafted the manuscript. MMH wrote the manuscript, JZ and XCL checked the manuscript. WQM revised and finalised the study protocol. All authors contributed to drafting the manuscript and have read and approved the final manuscript.

Funding This work was supported by the Jingdezhen City Hospital of Traditional Chinese Medicine and will be supported by the Jingdezhen City Hospital of Traditional Chinese Medicine in developing the protocol.

Competing interests None declared.

Patient consent Obtained.

Provenance and peer review Not commissioned; externally peer reviewed.

Open Access This is an Open Access article distributed in accordance with the Creative Commons Attribution Non Commercial (CC BY-NC 4.0) license, which permits others to distribute, remix, adapt, build upon this work non-commercially, and license their derivative works on different terms, provided the original work is properly cited and the use is non-commercial. See: http://creativecommons.org/ licenses/by-nc/4.0/

(C) Article author(s) (or their employer(s) unless otherwise stated in the text of the article) 2018. All rights reserved. No commercial use is permitted unless otherwise expressly granted.

\section{REFERENCES}

1. NIH Consensus Development Panel on Osteoporosis Prevention, Diagnosis, and Therapy. Osteoporosis prevention, diagnosis, and therapy. JAMA 2001;285:785-95. 
2. Burge $\mathrm{R}$, Dawson-Hughes $\mathrm{B}$, Solomon $\mathrm{DH}$, et al. Incidence and economic burden of osteoporosis-related fractures in the United States, 2005-2025. J Bone Miner Res 2007;22:465-75.

3. Poliakova I, Simakova ES, Sivordova LE, et al. [Osteoporosis in men: current issues]. Adv Gerontol 2015;28:77-9.

4. Masaryková L, Fulmeková M, Lehocká L', et al. [The quality of life of patients suffering from the osteoporosis]. Ceska Slov Farm 2015;64:72-8.

5. Tarantino U, Iolascon G, Cianferotti L, et al. Clinical guidelines for the prevention and treatment of osteoporosis: summary statements and recommendations from the Italian Society for Orthopaedics and Traumatology. J Orthop Traumatol 2017;18(Suppl 1):3-36.

6. Kotian P, Boloor A, Sreenivasan S. Study of adverse effect profile of parenteral zoledronic acid in female patients with osteoporosis. $J$ Clin Diagn Res 2016;10:0C04-6.

7. Paci M, Burks S, Wang MY. Consensus guidelines for the treatment of osteoporosis. Neurosurgery 2018;82:N6-N7.

8. McMillan LB, Zengin A, Ebeling PR, et al. Prescribing physical activity for the prevention and treatment of osteoporosis in older adults. Healthcare 2017;5:85

9. Simkin B. Even frail elderly patients can benefit from exercise. Geriatric Times 2002;3:1-5.

10. Yamazaki S, Ichimura S, Iwamoto J, et al. Effect of walking exercise on bone metabolism in postmenopausal women with osteopenia/ osteoporosis. J Bone Miner Metab 2004;22:500-8.

11. Gómez-Cabello A, Ara I, González-Agüero A, et al. Effects of training on bone mass in older adults: a systematic review. Sports Med 2012;42:301-25.

12. Murtezani A, Nevzati A, Ibraimi Z, et al. The effect of land versus aquatic exercise program on bone mineral density and physical function in postmenopausal women with osteoporosis: a randomized controlled trial. Ortop Traumatol Rehabil 2014;16:319-25.

13. Chen KM, Hsu YC, Chen WT, et al. Well-being of institutionalized elders after Yang-style Tai Chi practice. J Clin Nurs 2007;16:845-52.

14. Chan K, Qin L, Lau M, et al. A randomized, prospective study of the effects of Tai Chi Chun exercise on bone mineral density in postmenopausal women. Arch Phys Med Rehabil 2004;85:717-22.

15. Qin L, Choy W, Leung K, et al. Beneficial effects of regular Tai Chi exercise on musculoskeletal system. J Bone Miner Metab 2005;23:186-90.

16. Song $\mathrm{R}$, Lee EO, Lam $\mathrm{P}$, et al. Effects of Tai chi exercise on pain, balance, muscle strength, and perceived difficulties in physical functioning in older women with osteoarthritis: a randomized clinical trial. J Rheumatol 2003;30:2039-44.

17. Maciaszek J, Osiński W, Szeklicki R, et al. Effect of Tai Chi on body balance: randomized controlled trial in men with osteopenia or osteoporosis. Am J Chin Med 2007;35:1-9.

18. Song R, Roberts BL, Lee E-O, et al. A randomized study of the effects of Tai Chi on muscle strength, bone mineral density, and fear of falling in women with osteoarthritis. $J$ Altern Complement Med 2010;3:227-33.

19. Wayne PM, Kiel DP, Krebs DE, et al. The effects of Tai Chi on bone mineral density in postmenopausal women: a systematic review. Arch Phys Med Rehabil 2007;88:673-80.

20. Lee MS, Pittler MH, Shin BC, et al. Tai chi for osteoporosis: a systematic review. Osteoporos Int 2008;19:139-46.

21. Chang TJ, Ting YT, Sheu SL, et al. [Effects of tai chi in postmenopausal women with osteoporosis: a systematic review]. Hu Li Za Zhi 2014;61:75-84.

22. Shamseer L, Moher D, Clarke M, et al. Preferred reporting items for systematic review and meta-analysis protocols (PRISMA-P) 2015: elaboration and explanation. BMJ 2015;349:97647.

23. Rabin R, de Charro F. EQ-5D: a measure of health status from the EuroQol Group. Ann Med 2001;33:337-43.

24. Qu B, Guo HQ, Liu J, et al. Reliability and validity testing of the SF-36 questionnaire for the evaluation of the quality of life of Chinese urban construction workers. J Int Med Res 2009;37:1184-90.

25. Moher D, Liberati A, Tetzlaff J, et al. Preferred reporting items for systematic reviews and meta-analyses: the PRISMA statement. Int J Surg 2010;8:336-41.

26. Higgins JPT, Green S, eds. Cochrane handbook for systematic reviews of interventions version 5.3.0: The Cochrane Collaboration, 2015. http://handbook-5-3.cochrane.org/ (updated Oct 2015).

27. Lau J, loannidis JP, Schmid CH. Quantitative synthesis in systematic reviews. Ann Intern Med 1997;127:820-6.

28. Higgins JP, Thompson SG, Deeks JJ, et al. Measuring inconsistency in meta-analyses. BMJ 2003;327:557-60.

29. Higgins JP, Thompson SG. Quantifying heterogeneity in a metaanalysis. Stat Med 2002;21:1539-58.

30. Moher D, Liberati A, Tetzlaff J, et al. Preferred reporting items for systematic reviews and meta-analyses: the PRISMA statement. PLoS Med 2009;6:e1000097.

31. Atkins D, Best D, Briss PA, et al. GRADE Working Group. Grading quality of evidence and strength of recommendations. BMJ 2004;328:1490. 